\title{
Field deployment of loop-mediated isothermal amplification for centralized mass-screening of asymptomatic malaria in Zanzibar: a pre-elimination setting
}

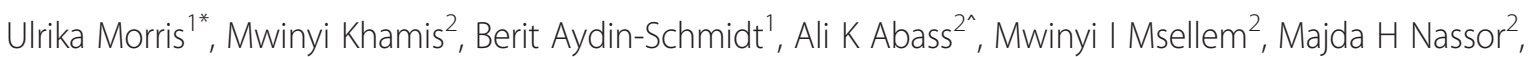
Iveth J González ${ }^{3}$, Andreas Mårtensson 1,4,5, Abdullah S Ali², Anders Björkman and Jackie Cook ${ }^{1,6}$

\begin{abstract}
Background: Molecular tools for detection of low-density asymptomatic Plasmodium infections are needed in malaria elimination efforts. This study reports results from the hitherto largest implementation of loop-mediated isothermal amplification (LAMP) for centralized mass screening of asymptomatic malaria in Zanzibar.

Methods: Healthy individuals present and willing to participate in randomly selected households in 60 villages throughout Zanzibar were screened for malaria by rapid diagnostic tests (RDT). In $50 \%$ of the study households, participants were asked to provide $60 \mu \mathrm{L}$ of finger-prick blood for additional LAMP screening. LAMP was conducted in two centralized laboratories in Zanzibar, by trained technicians with limited or no previous experience of molecular methods. The LAMP assay was performed with Loopamp ${ }^{\text {TM }}$ MALARIA Pan/Pf Detection Kit (Eiken Chemical Company, Japan). Samples positive for Plasmodium genus (Pan)-LAMP were re-tested using Plasmodium falciparum-specific LAMP kits.

Results: Paired RDT and LAMP samples were available from 3983 individuals. The prevalence of asymptomatic malaria was $0.5 \%$ (Cl $95 \%$ 0.1-0.8) and $1.6 \%$ (Cl 95 \% 1.1-2.2) by RDT and Pan-LAMP, respectively. LAMP detected 3.4 (Cl 95 \% 2.2-5.2) times more Plasmodium positive samples than RDT. DNA contamination was experienced, but solved by repetitive decontamination of all equipment and reagents.

Conclusions: LAMP is a simple and sensitive molecular tool, and has potential in active surveillance and mass-screening programmes for detection of low-density asymptomatic malaria in pre-elimination settings. However, in order to deploy LAMP more effectively in field settings, protocols may need to be adapted for processing larger numbers of samples. A higher throughput, affordable closed system would be ideal to avoid contamination.
\end{abstract}

Keywords: Plasmodium, Malaria, Low-density, Asymptomatic, Loop-mediated isothermal amplification, Mass screening, DNA contamination

\section{Background}

Asymptomatic Plasmodium infections are an important reservoir for continued malaria transmission that needs to be addressed in the context of malaria elimination [1]. Detection of asymptomatic infections, which are often sub-patent, i.e., fall beneath the threshold of detection of

\footnotetext{
* Correspondence: ulrika.morris@ki.se

Deceased

'Malaria Research, Department of Microbiology, Tumor and Cell Biology, Karolinska Institutet, Nobelsväg 16, 17177 Stockholm, Sweden

Full list of author information is available at the end of the article
}

both microscopy and rapid diagnostic tests (RDT), requires highly sensitive molecular tools. The use of polymerase chain reaction (PCR)-based assays in field settings is, however, limited due to the need for a cold chain, specialized equipment and know-how [2]. Loopmediated isothermal amplification (LAMP) offers several advantages over PCR in field settings. LAMP requires minimal equipment, has short time-to-result (30 min-1 h), with an analytical sensitivity similar to PCR, and results that can be read by eye using UV fluorescence [3-5].

\section{Ciomed Central}

(c) 2015 Morriset al.; licensee BioMed Central. This is an Open Access article distributed under the terms of the Creative Commons Attribution License (http://creativecommons.org/licenses/by/4.0), which permits unrestricted use, distribution, and reproduction in any medium, provided the original work is properly credited. The Creative Commons Public Domain Dedication waiver (http://creativecommons.org/publicdomain/zero/1.0/) applies to the data made available in this article, unless otherwise stated. 
The Loopamp ${ }^{\text {Tm }}$ MALARIA Pan/Pf Detection Kit (Eiken Chemical Company, Japan) has been developed as a field-friendly kit, comprising strips of reaction tubes containing vacuum-dried and temperature-stable reaction components for either Plasmodium genus (Pan)-specific or Plasmodium falciparum-specific malaria detection. The kit has been evaluated both in laboratory and field settings [6-8], and was piloted on a small scale in Zanzibar as a health facility-based, point-of-care, diagnostic tool for mass screening and treatment in 2013 [9].

This study reports results from the hitherto largest implementation of LAMP in the field, for scaled-up, centralized mass screening of asymptomatic malaria in Zanzibar, a pre-elimination setting.

\section{Methods}

\section{Study sites and study design}

Zanzibar, located $35 \mathrm{~km}$ off the coast of mainland Tanzania, consists of two main islands, Unguja and Pemba, with respective populations of approximately 900,000 and 400,000. This study was performed as part of a larger knowledge, attitude, practice, and behaviour (KAPB) malaria survey, conducted in Zanzibar April-May 2014. Household visits were carried out in 60 villages in ten districts (six in Unguja and four in Pemba) covering the whole of Zanzibar. A proportional number of households were sampled from each village to reach a sample size of 2162 households, powered for the KAPB study. Healthy individuals present and willing to participate in the randomly selected households were screened for malaria by RDT. In $50 \%$ of study households (in even house numbers), participants were asked to provide $60 \mu \mathrm{L}$ of finger-prick blood for additional LAMP screening. Nexus seven tablet computers were used to conduct questionnaires as part of the KAPB survey. All participants or guardians provided written informed consent prior to blood sampling. Ethical approvals were obtained from the ethical committees in Zanzibar (ZAMREC/0002/FEBRUARY/014) and the Regional Ethics Committee in Stockholm (2009/ 387-31).

\section{Training of field enumerators and sample collection}

Household visits were conducted by 40 field enumerators in teams of two, together with four field supervisors with prior experience of similar studies. All enumerators attended five days of training for RDT performance, blood sample collection for LAMP, and use of tablet computers. There were 14 teams in Unguja and six teams in Pemba, and each team visited six or seven households per day. RDT screening was conducted with either SD-Bioline Malaria Ag P.f/Pan ${ }^{\circledR}$ (Standard Diagnostic, Inc, USA) (used for $>90 \%$ of screening) or First Response ${ }^{\circledR}$ Malaria Ag Combo (pLDH/HRP2) (Premier Medical Corporation Limited India). Results were recorded on the tablet computer during household visits, and RDT positive individuals were referred to the closest health facility for treatment and registration in the Zanzibar malaria surveillance system. In $50 \%$ of study households, $60 \mu \mathrm{L}$ of finger-prick blood was collected using a plastic capillary tube (Dropstir, Medical Precision Plastics, USA), dispensed into a $1.5-\mathrm{ml}$ pre-labelled sample collection tube containing $60 \mu \mathrm{L}$ of pre-aliquoted DNA extraction buffer $(400 \mathrm{nM} \mathrm{NaCl}$, $40 \mathrm{mM}$ Tris pH 6.5, 0.45 SDS), and mixed by flicking. Blood samples were collected in microtube storage racks with lids and transported at the end of each day to two centralized laboratories, one on each island, where they were stored at $4{ }^{\circ} \mathrm{C}$ overnight.

\section{Training of laboratory technicians}

Four technicians, two for each laboratory, with limited or no experience of LAMP were trained over three-anda-half days. Training included a theoretical introduction to LAMP and the LAMP protocol, hands-on practical sessions with malaria positive blood samples diluted to different known concentrations, how to record results on tablet computers, and a half-day field trial with samples collected the same day by the field enumerators.

\section{Screening by LAMP in centralized laboratories}

LAMP procedures were similar to the pilot study [9], with some modifications for scale-up of sample sizes. One centrifuge, three heat-blocks $\left(1.5-\mathrm{ml}\right.$ block at $95{ }^{\circ} \mathrm{C}, 0.2-\mathrm{ml}$ block at $65^{\circ} \mathrm{C}$ and a $0.2-\mathrm{ml}$ block at $95^{\circ} \mathrm{C}$ ) and a UV lamp were required in each laboratory. All samples collected in Pemba and half of the samples collected in Unguja (see below) were processed within $24 \mathrm{~h}$ of sampling. To reduce the risk of mix-up of samples and contamination, sets of pre-labelled sample collection tubes (containing $60 \mu \mathrm{L}$ of aliquoted DNA extraction buffer) and pre-labelled DNA dilution tubes (containing $300 \mu \mathrm{L}$ of aliquoted sterile water) were prepared prior to the start of the study. DNA extraction and the LAMP assays were performed in separate areas to avoid contamination. DNA was extracted by the boil and spin method [10] and $26 \mu \mathrm{L}$ of the supernatant was transferred to the DNA dilution tubes. The LAMP assay was performed with Loopamp ${ }^{\text {tw }}$ MALARIA Pan/Pf Detection Kit (Eiken Chemical Company) as per protocol [10]. Samples positive for Pan-LAMP were retested using $P$. falciparum-LAMP specific kits. LAMP positive individuals (who were not positive by RDT) were visited by malaria surveillance officers and provided treatment within $48 \mathrm{~h}$ of sampling where possible.

\section{Freezing of samples}

Due to a delay in the shipment of LAMP kits, half of the LAMP samples collected in Unguja $(N=1414)$ were stored at $-20{ }^{\circ} \mathrm{C}$ after DNA extraction and dilution, until the remaining reaction tubes arrived five weeks later. 
Dilution tubes from LAMP-positive samples in Unguja were also stored at $-20{ }^{\circ} \mathrm{C}$, for quality control of frozen DNA.

\section{Statistical analysis}

Results are reported from individuals for which both RDT and LAMP were conducted (i.e., where paired data are available). Statistical analyses were conducted using Stata/SE 12.1 (StataCorp LP, Texas, USA). The survey design was taken into consideration when calculating $95 \%$ confidence intervals (CI $95 \%$ ) for prevalence estimations, using the survey [svy] command in Stata accounting for household and village sampling/stratification. The sensitivity and specificity of RDT was calculated using LAMP as the gold standard. McNemar's test was used to compare the methods. Statistical significance was determined as $p<0.05$.

\section{Results}

\section{Study population}

Participation was high; informed consent was given by $96.9 \%$ of those present at the time of the survey (Fig. 1). Both RDT and LAMP results were available for 3983/4085 $(97.5 \%)$ of the individuals willing to participate. The remaining $102(2.5 \%)$ were excluded from further analysis. The study population consisted of all ages (median: 18 years, range 0-98), with a higher proportion of females (59.0\%). Sample collection was conducted during a total of 19 days with an average of 220 samples processed per day in the two laboratories combined.

\section{Prevalence of malaria by RDT and LAMP}

The prevalence of asymptomatic malaria was $0.5 \%$ (CI $95 \%$ 0.1-0.8) and $1.6 \%$ (CI $95 \%$ 1.1-2.2) by RDT and Pan-LAMP, respectively (Table 1 ). Pan-LAMP detected 3.4 (CI 95 \% 2.2-5.2) times more Plasmodium positive samples than RDT. Out of the Pan-LAMP positive samples $64.6 \%(42 / 65)$ were also positive by $P$. falciparumLAMP. RDT had a sensitivity of $24.6 \%$ (14.7-36.9) and specificity of $99.9 \%$ (99.7-100.0) when compared to PanLAMP. Comparison by McNemar's test showed a significant difference between the two methods $(p<0.001)$.

\section{Discrepancies in LAMP after freezing of samples}

DNA extracted from half of the samples $(N=1414)$ in Unguja was stored at $-20{ }^{\circ} \mathrm{C}$ prior to LAMP testing due to a delay in the shipment of LAMP kits. Among these samples, 32 (2.3\%) were positive by Pan-LAMP, out of which 12 was also positive by RDT. However, amongst the frozen samples there were also three RDT positive samples that were found negative by Pan-LAMP. These three samples were positive for $P$. falciparum HRP2 only, Pan-Plasmodium LDH only, and both P. falciparum HRP2 and Pan-Plasmodium LDH, respectively. Among the samples from Unguja that were screened before freezing $(N=1370), 11(0.8 \%)$ were positive by PanLAMP, out of which one was also positive by RDT. The 11 Pan-LAMP positive samples were stored at $-20{ }^{\circ} \mathrm{C}$, as a quality control of freezing DNA, however only $7 / 11$ $(63.6 \%)$ were positive when re-tested after thawing.

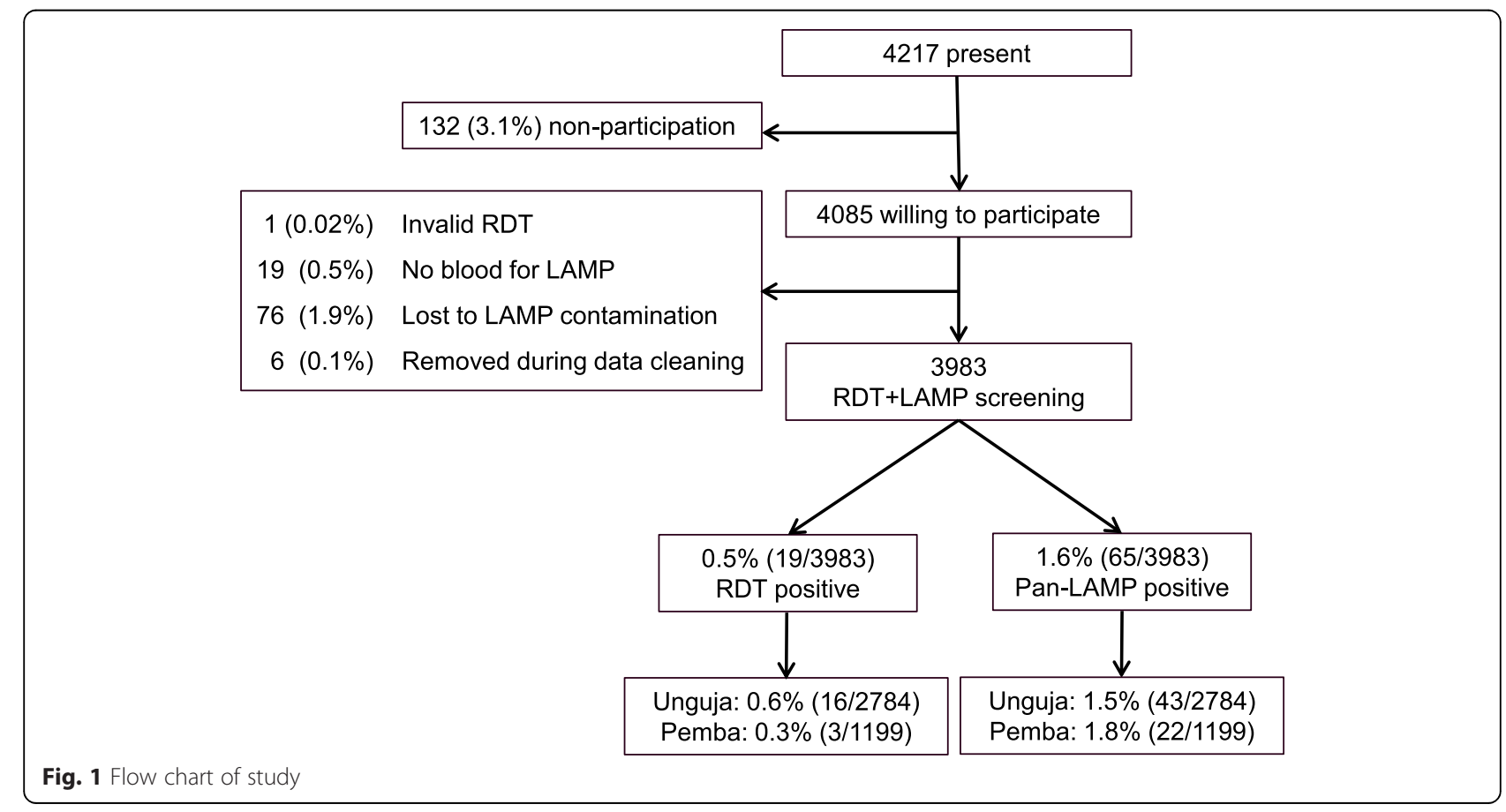


Table 1 Prevalence of malaria detected by RDT and LAMP

\begin{tabular}{|c|c|c|}
\hline & RDT & LAMP \\
\hline \multirow[t]{2}{*}{ Overall prevalence $\left(\% ; \mathrm{Cl} 95 \%{ }^{\mathrm{a}}\right)$} & $0.5 ; 0.1-0.8$ & $1.6 ; 1.1-2.2$ \\
\hline & 19/3983 & $65 / 3983$ \\
\hline \multicolumn{3}{|l|}{ Relative proportion positive in: } \\
\hline \multirow[t]{2}{*}{ Only Pan ${ }^{\mathrm{b}} \% ; \mathrm{Cl} 95 \%$ ) } & $5.3 ; 0.0-16.4$ & $35.4 ; 23.4-47.4$ \\
\hline & $1 / 19$ & $23 / 65$ \\
\hline \multirow[t]{2}{*}{ Pan + P. falciparum ${ }^{c}(\% ;$ Cl $95 \%)$} & $31.6 ; 8.5-54.6$ & $64.6 ; 52.6-76.6$ \\
\hline & $6 / 19$ & $42 / 65$ \\
\hline \multirow[t]{2}{*}{ Only P. falciparum (\%; Cl $95 \%)$} & $63.2 ; 39.2-87.1$ & $N D^{e}$ \\
\hline & $12 / 19$ & \\
\hline
\end{tabular}

Both RDT brands used for malaria screening are two-band RDTs detecting P. falciparum HRP2 and Pan-Plasmodium LDH simultaneously, although with different detection limits (50-100 parasites/ $\mu \mathrm{L}$ for $P$. falciparum HRP2 and 200-500 parasites/ $\mu \mathrm{L}$ for Pan-Plasmodium LDH). In contrast, only the Pan-LAMP positive samples were assessed for $P$. falciparum during the LAMP screening, with a detection limit of 2-5 parasites $/ \mu \mathrm{L}$ for both Pan-Plasmodium and $P$. falciparum

${ }^{a}$ Confidence intervals for prevalences were calculated using the survey [svy] command in Stata, accounting for household and village sampling/stratification

${ }^{\mathrm{b}}$ Positive for Plasmodium genus only

'Positive for Plasmodium and P. falciparum

${ }^{\mathrm{d}}$ Positive for $P$. falciparum only

${ }^{\mathrm{e}} \mathrm{ND}=$ not determined

\section{LAMP-amplified DNA contamination}

During the study DNA contamination of LAMP arose in the central laboratory in Pemba [see Additional file 1 for flow chart of events]. The contamination resulted from using a heat block with a heated pressurized lid during the $95{ }^{\circ} \mathrm{C}$ enzyme inactivation stage, and not allowing the samples to cool to room temperature before removing the strips for recording of results. 'Fizzing' was observed around the lid of the LAMP strips resulting in leakage of LAMP-amplified DNA. All equipment and reagents were subjected to repetitive decontamination with $5 \%$ sodium hypochlorite over three days, and moved away from the epicentre of the contamination to a laboratory space available in another building. The final enzyme inactivation step of the protocol [10] was removed as this was thought to be the source of contamination; instead results were read and recorded immediately after the amplification reaction. A negative control was included in each strip of eight reaction tubes, and any Pan-LAMP positive samples were repeated and only recorded as positive if positive in both runs. During the first few days following the contamination there were some samples that were considered false positive, but the numbers declined and reached zero within one week after the contamination.

\section{Discussion}

This is the hitherto largest reported implementation of LAMP for detection of asymptomatic malaria in a field setting. In order to scale-up the breadth of sampling, LAMP testing was centralized in two laboratories, meaning samples could be collected from all over the islands with fewer resources. The time-to-result was approximately $24 \mathrm{~h}$, compared with three hours in the pilot study where LAMP was used as a health facility-based, point-of-care, diagnostic tool for mass screening and treatment [9].

The results confirm the improved sensitivity of LAMP over RDT, as has been shown previously [3, 9]. The MALARIA Pan/Pf Detection Kit has a detection limit of 2-5 parasites/ $\mu \mathrm{L}[3,6]$, for both Pan-Plasmodium and $P$. falciparum. This is comparable to PCR, and substantially lower than the detection limits of $P$. falciparum-specific HRP2 (50-100 parasites/ $\mu \mathrm{L}$ ) and Pan-Plasmodium LDH (200-500 parasites/ $\mu \mathrm{L}$ ) in combo RDTs. The proportion of samples detected only by Pan-LAMP (35.4\%) suggests the presence of species other than P. falciparum. Similarly, other studies in Zanzibar have shown that up to $40 \%$ of PCR-detectable malaria infections contained non-falciparum species $[11,12]$. Non-falciparum infections tend to be of lower parasite densities than $P$. falciparum infections [13], emphasizing the need for more sensitive species-specific methods for non-falciparum Plasmodium detection. The sensitivity (83.8 \%) and specificity (99.7\%) of Pan-LAMP, calculated using PCR as the reference standard, was high in the pilot study conducted in Zanzibar [9]. This is similar to previously reported sensitivities and specificities $[6-8,14,15]$ and, together with the results of this study, suggests malaria LAMP is a useful molecular tool sensitive enough for detection of lowdensity asymptomatic malaria infections in field settings.

Importantly, some discrepancies were shown amongst samples screened following freezing of diluted DNA. RDT false positivity due to recently cleared infections has been well documented when detecting $P$. falciparum HRP2 [16], although none of the three study participants who were RDT positive/LAMP negative reported receiving malaria treatment within the previous two weeks, and two of the RDTs were positive for Pan-Plasmodium LDH suggesting ongoing infections. The lack of reproducibility of results following freezing of samples suggests that DNA extracted by simple methods such as boil and spin may not be suitable for long-term storage and should be amplified by LAMP within a short period of time [17]. Alternatively, low reproducibility of PCR for detection of low-density infections has been reported [18] and parasite densities close to the LAMP detection limit could also explain the lack of reproducibility.

The potential risk of contamination with LAMP is large, due to the high efficiency of the reaction, although the risk is reduced when using a closed system $[3,19]$. The MALARIA Pan/Pf Detection Kit is manufactured with tubes that cannot be re-opened once closed, in order to avoid contamination with amplified DNA. However, as demonstrated in this study, the exposure of such tubes to high 
temperatures, as during enzyme inactivation, results in softening of the plastic and leakage of the contents. While removing the inactivation step solved this problem in this case, contaminations have been experienced in other research settings $[8,20]$ and these issues are important to report. Although MALARIA Pan/Pf Detection Kit is a field-friendly option, three days' training is not sufficient for dealing with such events. Successful decontamination requires a larger understanding of molecular techniques and rigorous repetitive methods to ensure that the area is free of contamination.

Standard malaria diagnostic tools including microscopy and RDT are not sensitive enough to detect low-density asymptomatic infections [12]. Nucleic acid amplificationbased methods provide the, to date, most sensitive and accurate tools to detect and identify pathogens [21]. Recently published, highly sensitive quantitative PCR methods state detection limits as low as 0.02 and 0.03 parasites $/ \mu \mathrm{L}$ blood [22, 23]. However, these methods lack the field applicability that LAMP offers. Furthermore, the cost of LAMP is estimated to be a tenth of that of conventional PCR [15], although the cost of the field friendly kit is still at 5.3 US\$ per reaction i.e., considerably more expensive than RDTs [3].

The high cost and risk of contamination may yet limit the implementation LAMP at a point-of-care level, but LAMP will be valuable for research purposes and for evaluating malaria elimination efforts. LAMP may, for example, be useful in mass/focal screening and treatment (MSAT/FSAT) programmes, for which the deployment of RDTs, perhaps due to their low sensitivity, has had varying results $[11,24]$. In any case it will be important to evaluate the impact and cost effectiveness of deploying LAMP, in comparison to the deployment of standard diagnostic tools as well as in comparison to alternative molecular methods.

\section{Conclusions}

LAMP is a simple and sensitive molecular tool, and has potential in active surveillance and mass-screening programmes for detection of low-density asymptomatic malaria in pre-elimination settings. However, in order to deploy LAMP more effectively in field settings, protocols may need to be adapted for processing larger numbers of samples. A higher throughput, affordable closed system would be ideal to avoid contamination.

\section{Additional file}

Additional file 1: Flow chart of events that took place during LAMP DNA contamination and decontamination. A figure showing the flow of events that took place during a DNA contamination when screening for asymptomatic malaria by LAMP in Zanzibar The flow chart shows how the DNA contamination was resolved by repetitive decontamination of all equipment with $5 \%$ sodium hypochlorite and moving away from the site of contamination.

\section{Competing interests}

$I G$ is an employee of the Foundation for Innovative New Diagnostics (FIND), a co-developer of the Loopamp ${ }^{\text {TM }}$ MALARIA Pan/Pf Detection Kit. All other authors declare no competing interest.

\section{Authors' contributions}

MIM, IJG, AM, ASA, AB, and JC conceived and designed the study. UM, MK, $B A S, A K A$, and JC carried out the training and were responsible for the fieldwork in Zanzibar. UM, BAS and MHN were responsible for the training and conducting of LAMP. UM and JC analysed the data, and drafted the manuscript. All authors read and approved the final manuscript.

\section{Acknowledgments}

We would like to thank all participants, staff members, and ZAMEP employees involved in the KAPB survey for their dedicated participation. We would also like to acknowledge Colin Sutherland, Spencer Polly, Michelle Hsiang and Alanna Schwartz for their intellectual input regarding experience of dealing with DNA contamination. This work was supported by Global Fund [Grant number ZAN-809-G07-M, work plan GFRD 8 phase 2 2013]; President's Malaria Initiative (PMI) [Implementation letter \# 45, work plan for FY 2013]; the Swedish Medical Research Council (VR) [grant numbers 2009-3785 and 2013-6594]; the Foundation for Innovative New Diagnostics (FIND) with funds from the German Federal Ministry of Education and Research (BMBF) through the KfW Entwicklungsbank; and the Einhorn foundation. In memoriam of Ali K Abass, a much missed friend and colleague.

\section{Author details}

${ }^{1}$ Malaria Research, Department of Microbiology, Tumor and Cell Biology, Karolinska Institutet, Nobelsväg 16, 17177 Stockholm, Sweden. ²Zanzibar Malaria Elimination Programme (ZAMEP), Ministry of Health, Zanzibar, Tanzania. ${ }^{3}$ Foundation for Innovative New Diagnostics (FIND), Geneva, Switzerland. ${ }^{4}$ Department of Public Health Sciences, Karolinska Institutet, Stockholm, Sweden. ${ }^{5}$ Centre for Clinical Research Sörmland, Uppsala University, Uppsala, Sweden. 'ondon School of Hygiene and Tropical Medicine, London, UK.

Received: 23 March 2015 Accepted: 8 May 2015

Published online: 17 May 2015

\section{References}

1. Bousema T, Okell L, Felger I, Drakeley C. Asymptomatic malaria infections: detectability, transmissibility and public health relevance. Nat Rev Microbiol. 2014;12:833-40.

2. Hanscheid T, Grobusch MP. How useful is PCR in the diagnosis of malaria? Trends Parasitol. 2002;18:395-8.

3. Han ET. Loop-mediated isothermal amplification test for the molecular diagnosis of malaria. Expert Rev Mol Diagn. 2013;13:205-18.

4. Oriero EC, Jacobs J, Van Geertruyden JP, Nwakanma D, D'Alessandro U. Molecular-based isothermal tests for field diagnosis of malaria and their potential contribution to malaria elimination. J Antimicrob Chemother. 2014;70:2-13.

5. Notomi T, Okayama H, Masubuchi H, Yonekawa T, Watanabe K, Amino N, et al. Loop-mediated isothermal amplification of DNA. Nucleic Acids Res. 2000;28:E63

6. Aydin-Schmidt B, Xu W, Gonzalez IJ, Polley SD, Bell D, Shakely D, et al. Loop mediated isothermal amplification (LAMP) accurately detects malaria dna from filter paper blood samples of low density parasitaemias. PLoS One. 2014;9:e103905

7. Hopkins H, Gonzalez IJ, Polley SD, Angutoko P, Ategeka J, Asiimwe C, et al. Highly sensitive detection of malaria parasitemia in a malaria-endemic setting: performance of a new loop-mediated isothermal amplification kit in a remote clinic in Uganda. J Infect Dis. 2013;208:645-52.

8. Polley SD, Gonzalez IJ, Mohamed D, Daly R, Bowers K, Watson J, et al. Clinical evaluation of a loop-mediated amplification kit for diagnosis of imported malaria. J Infect Dis. 2013;208:637-44.

9. Cook J, Schmidt B, Gonzalez IJ, Bell D, Edlund E, Nassor MH, et al. Loop-mediated isothermal amplification (LAMP) for point-of-care detection of asymptomatic low-density malaria parasite carriers in Zanzibar. Malar J. 2015;14:43. 
10. FIND: Manual of standard operating procedures for malaria LAMP [http://www.finddiagnostics.org/export/sites/default/programs/malaria-afs/ docs/SOPs_LAMP_Malaria_AUG12.pdf]

11. Cook J, Xu W, Msellem M, Vonk M, Bergstrom B, Gosling R, et al. Mass screening and treatment using a Plasmodium falciparum-specific rapid diagnostic test did not reduce malaria incidence in Zanzibar. J Infect Dis. 2014;211:1476-83.

12. Morris U, Xu W, Msellem MI, Schwartz A, Abass A, Shakely D, et al. Characterising temporal trends in asymptomatic Plasmodium infections and transporter polymorphisms during transition from high to low transmission in Zanzibar, 2005-2013. Infect Genet Evol. 2015. doi:10.1016/j.meegid.2015.04.018.

13. Cotter C, Sturrock HJ, Hsiang MS, Liu J, Phillips AA, Hwang J, et al. The changing epidemiology of malaria elimination: new strategies for new challenges. Lancet. 2013;382:900-11.

14. Polley SD, Mori Y, Watson J, Perkins MD, Gonzalez IJ, Notomi T, et al. Mitochondrial DNA targets increase sensitivity of malaria detection using loop-mediated isothermal amplification. J Clin Microbiol. 2010:48:2866-71.

15. Poon LL, Wong BW, Ma EH, Chan KH, Chow LM, Abeyewickreme W, et al. Sensitive and inexpensive molecular test for falciparum malaria: detecting Plasmodium falciparum DNA directly from heat-treated blood by loop-mediated isothermal amplification. Clin Chem. 2006;52:303-6.

16. Mayxay M, Pukrittayakamee S, Chotivanich K, Looareesuwan S, White NJ. Persistence of Plasmodium falciparum HRP-2 in successfully treated acute falciparum malaria. Trans R Soc Trop Med Hyg. 2001:95:179-82.

17. Sattabongkot J, Tsuboi T, Han ET, Bantuchai S, Buates S. Loop-mediated isothermal amplification assay for rapid diagnosis of malaria infections in an area of endemicity in Thailand. J Clin Microbiol. 2014;52:1471-7.

18. Costa DC, Madureira AP, Amaral LC, Sanchez BA, Gomes LT, Fontes CJ, et al. Submicroscopic malaria parasite carriage: how reproducible are polymerase chain reaction-based methods? Mem Inst Oswaldo Cruz. 2014;109:21-8.

19. Hsiang MS, Greenhouse B, Rosenthal PJ. Point of care testing for malaria using LAMP, loop mediated isothermal amplification. J Infect Dis. 2014:210:1167-9.

20. Paris DH, Imwong M, Faiz AM, Hasan M, Yunus EB, Silamut $K$, et al. Loop-mediated isothermal PCR (LAMP) for the diagnosis of falciparum malaria. Am J Trop Med Hyg. 2007;77:972-6.

21. Abdul-Ghani R, Al-Mekhlafi AM, Karanis P. Loop-mediated isothermal amplification (LAMP) for malarial parasites of humans: would it come to clinical reality as a point-of-care test? Acta Trop. 2012;122:233-40.

22. Hofmann N, Mwingira F, Shekalaghe S, Robinson LJ, Mueller I, Felger I. Ultra-sensitive detection of Plasmodium falciparum by amplification of multi-copy subtelomeric targets. PLoS Med. 2015;12:e1001788.

23. Imwong M, Hanchana S, Malleret B, Renia L, Day NP, Dondorp A, et al. High throughput ultra-sensitive molecular techniques to quantify low density malaria parasitaemias. J Clin Microbiol. 2014;52:3303-9.

24. Sutcliffe CG, Kobayashi T, Hamapumbu H, Shields T, Mharakurwa S, Thuma $\mathrm{PE}$, et al. Reduced risk of malaria parasitemia following household screening and treatment: a cross-sectional and longitudinal cohort study. PLoS One. 2012;7:e31396

\section{Submit your next manuscript to BioMed Central and take full advantage of:}

- Convenient online submission

- Thorough peer review

- No space constraints or color figure charges

- Immediate publication on acceptance

- Inclusion in PubMed, CAS, Scopus and Google Scholar

- Research which is freely available for redistribution

Submit your manuscript at www.biomedcentral.com/submit 